\title{
THz Arrays in CMOS
}

\author{
Patrick Reynaert \\ ESAT-MICAS, KU Leuven \\ Leuven, Belgium \\ patrick.reynaert@kuleuven.be
}

\author{
Kaizhe Guo \\ ESAT-MICAS, KU Leuven \\ Leuven, Belgium \\ kaizhe.guo@esat.kuleuven.be
}

\author{
Gabriel Guimaraes \\ ESAT-MICAS, KU Leuven \\ Leuven, Belgium \\ gabriel.teofilonevesguimaraes@kuleuven.be
}

\begin{abstract}
In this paper, two CMOS arrays above $0.5 \mathrm{THz}$ are introduced. By coupling numerous radiator elements together, state-of-the-art radiated power is achieved. At $0.59 \mathrm{THz}$, a coherent radiator array is designed in $40 \mathrm{~nm}$ CMOS. Combining 36 coherent radiators on $0.68 \mathrm{~mm}^{2}$, it achieves $1 \mathrm{~mW}$ radiated power, 24.1 dBm EIRP and 0.8\%o DC-to-THz efficiency. Further, a $0.67 \mathrm{THz}$ radiator array using a novel oscillator topology is introduced. The oscillator core benefits from a harmonic tank and a filtering feedback network to increase its harmonic power generation. The array includes 8 intercoupled oscillator cores. This design achieves $-16.1 \mathrm{dBm}$ radiated power and $7.4 \mathrm{dBm}$ EIRP in $40 \mathrm{~nm}$ CMOS.
\end{abstract}

Index Terms-THz, Arrays, CMOS, Harmonic Oscillator

\section{INTRODUCTION}

In a $\mathrm{THz}$ active imaging system, a signal source with high output power is required to provide illumination on the object [1]. Conventionally, $\mathrm{THz}$ integrated circuits are mainly designed using III-V technologies. However, III-V technologies are rather expensive and cannot be integrated with high-density digital circuits for signal process. Compared to III-V technologies, CMOS technologies have the advantage of low cost and full integration with digital circuits. Since the $\mathrm{THz}$ frequency range lies beyond $f_{\max }$ of CMOS transistors, harmonic extraction is needed to generate $\mathrm{THz}$ signals. As a result, the power from a single $\mathrm{THz}$ radiator is limited [2], [3]. To further increase the radiated power, a coherent radiator array with free-space power combining is needed [4]-[11]. As shown in Fig. 1, a coherent radiator array effectively increases the radiated power compared to single radiator.

In this paper, two CMOS arrays above $0.5 \mathrm{THz}$ are introduced. By composing radiator arrays with numerous intercoupled radiator elements, state-of-the-art radiated power is achieved. In Section II, the design and measurement of a $0.59 \mathrm{THz}$ coherent radiator array in $40 \mathrm{~nm}$ CMOS is introduced. In this array, a coupling structure with low impact on the radiator elements is proposed. Using this coupling structure, 36 radiators are coupled together to increase the radiated power. In Section III, a scalable $0.67 \mathrm{THz}$ radiator array is introduced. In this topology a compact oscillator cell simultaneously boosts the harmonic power generation, radiates the high-frequency signal off-chip and couples to adjacent cells to maintain phase coherency between oscillators. The session

This work was supported by the CHIST ERA project WISDOM under FWO Grant G0H5816N.

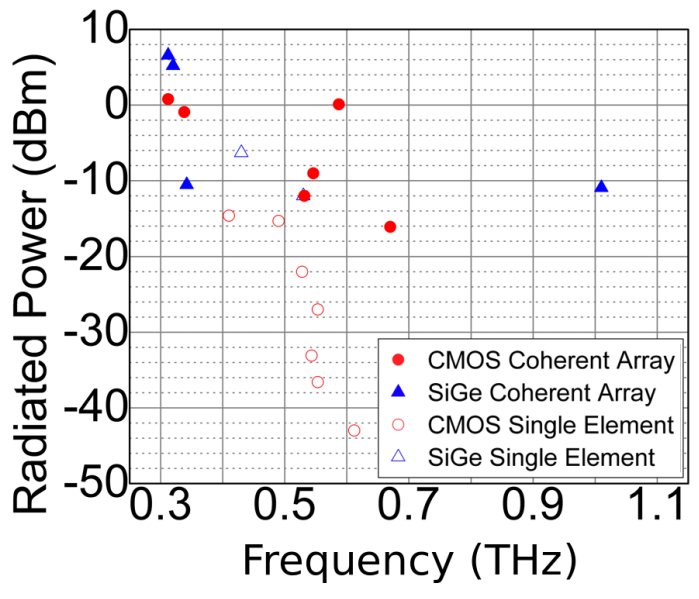

Fig. 1. Radiated power of silicon-based $\mathrm{THz}$ radiating source.

also presents the measured results of the manufactured chip prototype.

\section{A $0.59 \mathrm{THz} 6 \times 6$ COHERENT RADIATOR ARRAY IN 40 NM CMOS}

The first design is a $0.59 \mathrm{THz}$ radiator array in $40 \mathrm{~nm}$ CMOS. As shown in Fig. 2, the coherent array has 36 oscillator cores operating at $147 \mathrm{GHz}\left(f_{0}\right)$ which generate an in-phase signal at the fourth harmonic frequency of $0.59 \mathrm{THz}\left(4 f_{0}\right)$. Each oscillator core is a cross-coupled pair with microstrip line between the gate of one transistor and the drain of the other transistor. At $4 f_{0}$, this microstrip line transforms the low impedance at the gate to a high impedance at the drain, and stops the fourth harmonic power generated at the drain of one transistor going into the gate of the other transistor. The fourth harmonic power is delivered to the antenna through a capacitive L-type impedance matching network with compact layout. The DC supply network provides an open circuit at $4 f_{0}$. At $2 f_{0}$, it provides a reactance required formaximizing the fourth harmonic generation. The area of one oscillator core together with the antenna is $0.01 \mathrm{~mm}^{2}$.

CMOS technology allows metal lines with much smaller width and thickness compared to III-V technologies. This ability is utilized in this work to design a compact coupling structure in the array with extremely low impact on the oscillator core itself. As shown in Fig. 3, the coupling structure 


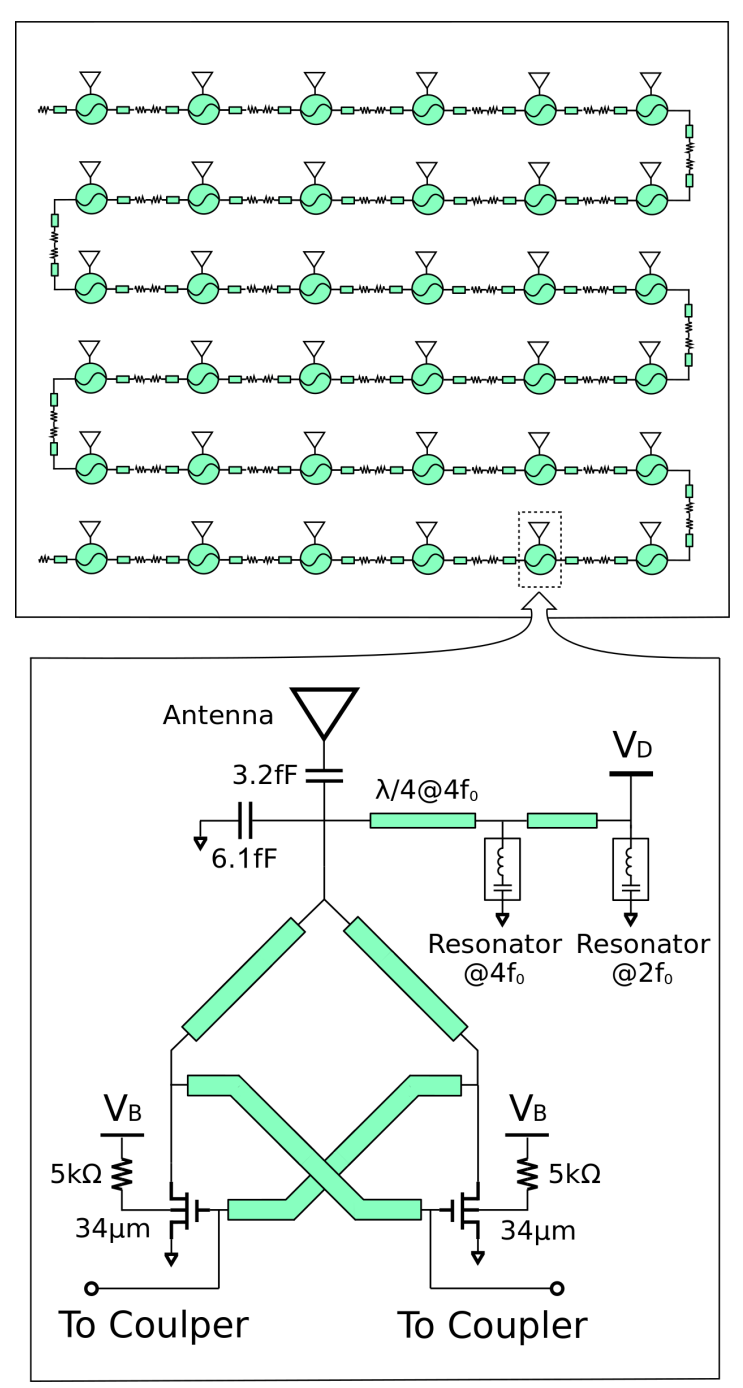

Fig. 2. Architecture of the $0.59 \mathrm{THz}$ coherent radiator array in $40 \mathrm{~nm}$ CMOS.

has a via-based resistor in the middle, and two $0.36 \mu \mathrm{m}$ microstrip lines on both sides connected to the gates of the transistors of adjacent oscillator cores. For differential-mode excitation, a virtual ground is created at the center of the coupling structure. The $24 \Omega$ via resistor is transformed to an impedance equivalent to a $50.8 \Omega$ resistor in parallel with a $66.4 \mathrm{pH}$ inductor at $f_{0}$. This parallel resistor stops any possible differential-mode oscillation. For common mode excitation, a virtual open is created at the center of the coupling structure. As the parasitic parallel capacitance of the via resistor is only $0.5 \mathrm{fF}$ and the width of the microstrip line is very narrow, the impedance of the coupling structure at $f_{0}$ is equivalent to a small capacitor in parallel with a big resistor (4.7 fF and $29 \mathrm{k} \Omega$ ). The small capacitor only contributes to $6.7 \%$ effective capacitance in the oscillator tank, and the big resistor has negligible impact on the overall quality of the oscillator tank. At $4 f_{0}$, the coupling structure shows an impedance of $(3.8-\mathrm{j} * 22) \Omega$ connected in parallel to the transistor gate. In simulation, only $0.1 \mathrm{~dB}$ reduction of the output power at $4 f_{0}$
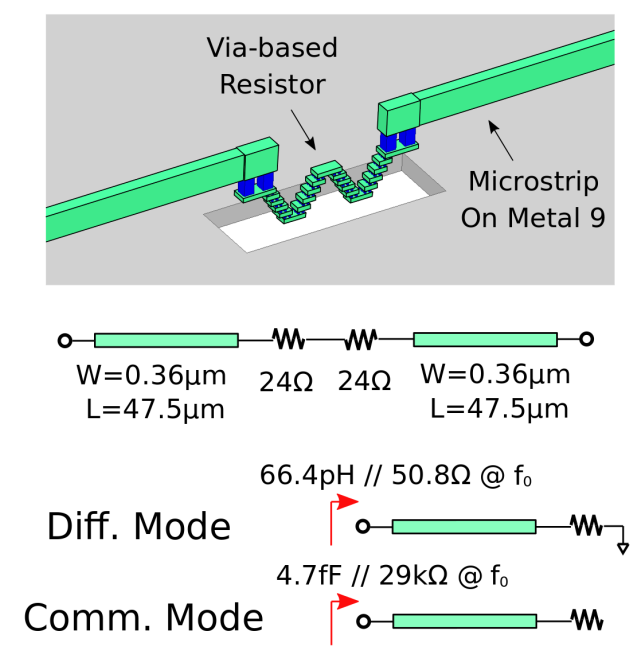

Fig. 3. The coupling structure used in the $0.59 \mathrm{THz}$ coherent radiator array.

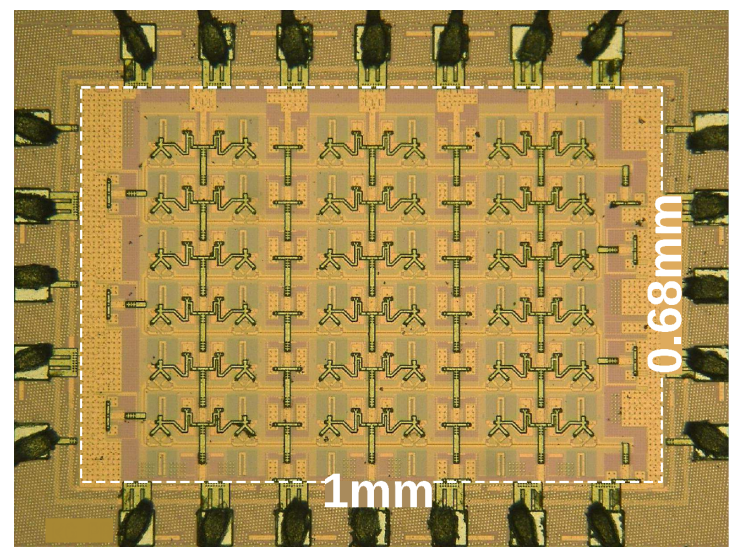

Fig. 4. Die micrograph of the $0.59 \mathrm{THz}$ coherent radiator array.

is caused due to the coupling structure. Two dummy coupling structures are connected to both edges of the array. The dummy coupling structure is half of the real coupling structure with a floating end point which can perfectly duplicate the virtual open in the real coupling structures, and will not cause any imbalance in the radiator array.

Figure 4 shows the die micrograph of the $0.59 \mathrm{THz}$ coherent radiator array. To measure the antenna directivity accurately, the 2-D radiation pattern of the array is measured and shown in Fig. 5. By integrating the measured 2-D radiation pattern, the directivity is calculated as $24 \mathrm{~dB}$. The radiated power for $0.9 \mathrm{~V} V_{D}$ is calculated as $0.1 \mathrm{dBm}$ using the measured EIRP and directivity. The power consumption of the chip for $0.9 \mathrm{~V}$ $V_{D}$ is $1.278 \mathrm{~W}$. Figure 6 also shows the EIRP and the output frequency of the array for different $V_{D}$.

\section{A $0.67 \mathrm{THZ} 4 \times 2$ RADIATOR ARRAY IN 40 NM CMOS}

The second design is a $4 \times 2$ oscillator array as shown in Fig. 7. The oscillators operate at a fundamental frequency of $223 \mathrm{GHz}$ with third harmonic extracted at $670 \mathrm{GHz}$. The 
$(\mathrm{dBi})$

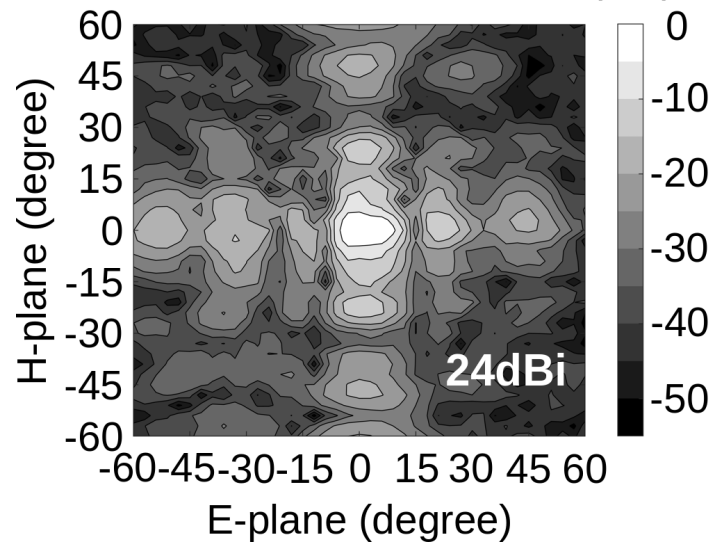

Fig. 5. Measured 2-D radiation pattern of the $0.59 \mathrm{THz}$ array.

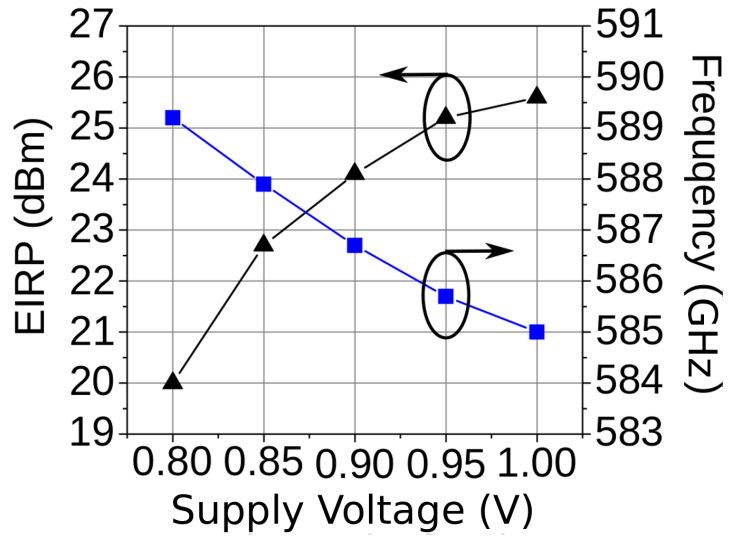

Fig. 6. Measured EIRP and the output frequency of the $0.59 \mathrm{THz}$ array for different $V_{D}$.

figure also depicts the different coupling modes between each cell, with some cells coupling in common-mode and some in differential-mode. As it will be explained later, this creates the necessary field distribution for efficient radiation in the cells, which are spaced in $\lambda / 2$ units for far-field coherent radiation. Also in the figure, metal walls are depicted. These metal walls are implemented in all metal and via layers of the technology. This emulates the presence of additional deferentially-coupled cells and thus reduce the mismatch in the oscillation frequency of the oscillators at the edge of the array when compared to those in the center of the array.

Figure 8 depicts the layout of a single differential oscillator cell. It consists of a folded slotline tank that provides the transistors with a high impedance resonance at both $f_{0}$ and $3 f_{0}$ and a low-impedance at $2 f_{0}$. It can be shown that these conditions are the optimal for boosting the generation of third harmonic current at the drain of the transistor [12]. A microstrip line is used to couple the signal back to the transistors gate and close the feedback loop. The lines couple at a virtual ground at the $3 f_{0}$, thus creating a filtering network.

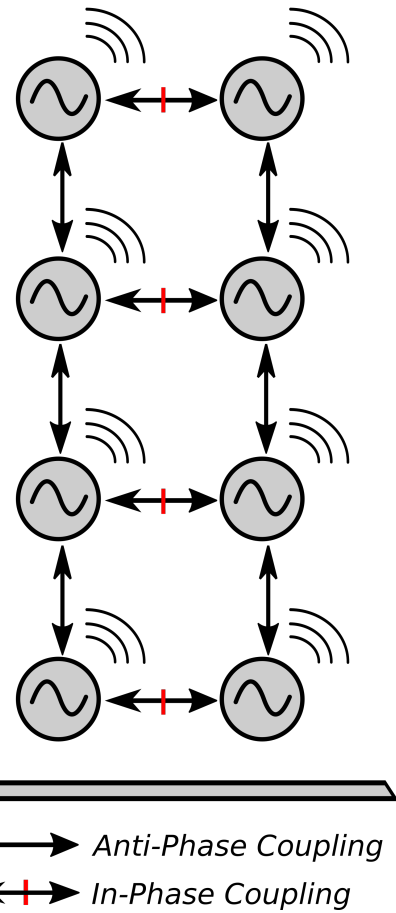

Fig. 7. Architecture of the $0.67 \mathrm{THz} 4 \times 2 \mathrm{THz}$ oscillator-radiator array highlighting phase in coupling between cells.

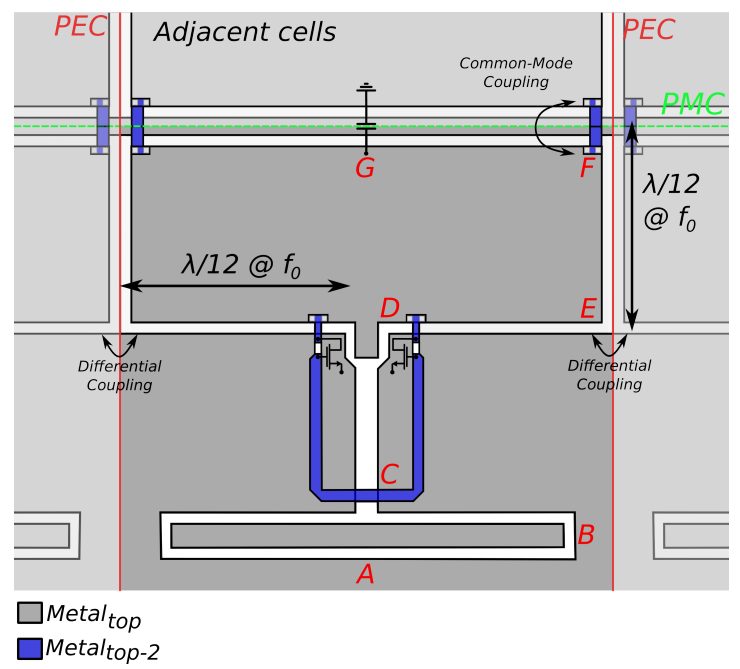

Fig. 8. Single-cell layout depicting slotline tank and feedback microstrip line. Coupling to adjacent cells are also depicted with equivalent metal walls (PEC/PMC) used for simulation.

This prevents the lossy gate node of loading the tank at the harmonic frequency, increasing the available power to be radiated by the slots.

The chip radiates the $\mathrm{THz}$ signal through the silicon substrate. The mechanism of radiation is depicted in Fig. 9. One can see that the E-Fields of two front-facing cells build up the same field pattern as a $\lambda / 2$ slot. This way, the whole source 


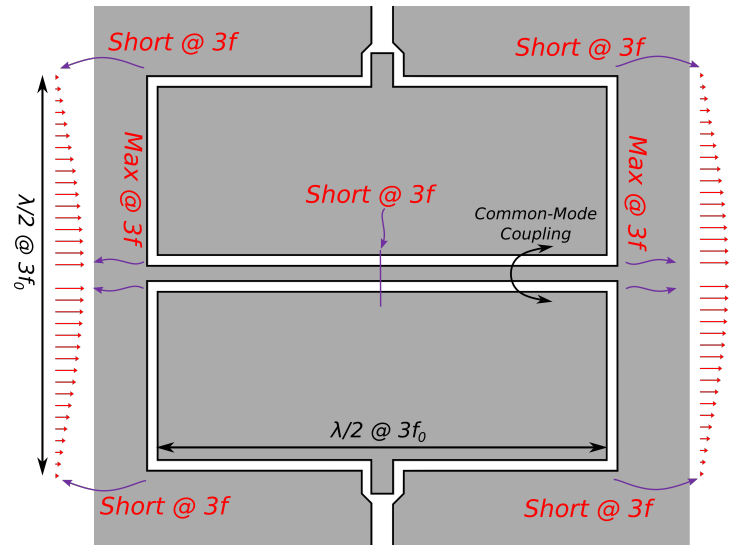

Fig. 9. Common-mode coupled cells tanks with field distribution at third harmonic. Fields build two $\lambda / 2$-spaced slotline antennas and radiate the signal off-chip.

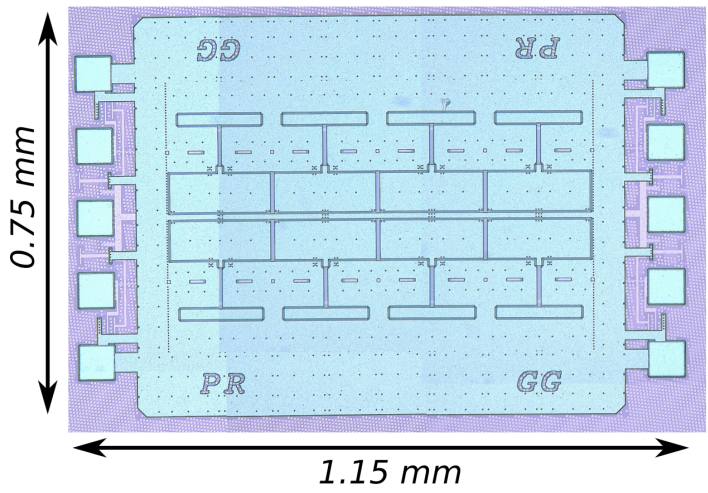

Fig. 10. Die micrograph of the $0.67 \mathrm{THz} 4 \times 2 \mathrm{THz}$ radiated array in $40 \mathrm{~nm}$ CMOS.

has a linear array of 5 slot-antennas. To increase the directivity of the structure a hyper-hemispherical lens is attached to the backside of the silicon substrate.

The design was implemented in a $40 \mathrm{~nm}$ CMOS technology. The chip micrograph can be seen in Fig. 10. The measured EIRP over the circuit tuning range is depicted in Figure 11. The maximum EIRP is $7.4 \mathrm{dBm}$ at $670 \mathrm{GHz}$. The radiation pattern is shown in Fig. 12. The peak directivity of the source was measured at $23.5 \mathrm{dBi}$, which implies a total radiated power of $-16.1 \mathrm{dBm}$ while consuming $99.7 \mathrm{~mW}$ of DC power.

\section{CONCLUSION}

In this paper, two CMOS arrays above $0.5 \mathrm{THz}$ are introduced. The first work is a $0.59 \mathrm{THz}$ coherent radiator array in $40 \mathrm{~nm}$ CMOS. By coupling 36 radiators, a state-of-theart radiated power is achieved. The second work, a $0.67 \mathrm{THz}$ radiator array, demonstrated that novel design techniques and circuit topologies can enable CMOS as a viable technology for a frequency range above $650 \mathrm{GHz}$ without compromising the power performance. 8 radiator cores are coupled in this array. The proposed array can be scaled to even larger sizes if applications require, due to the low DC power consumption and compact area. In both of the proposed arrays, the

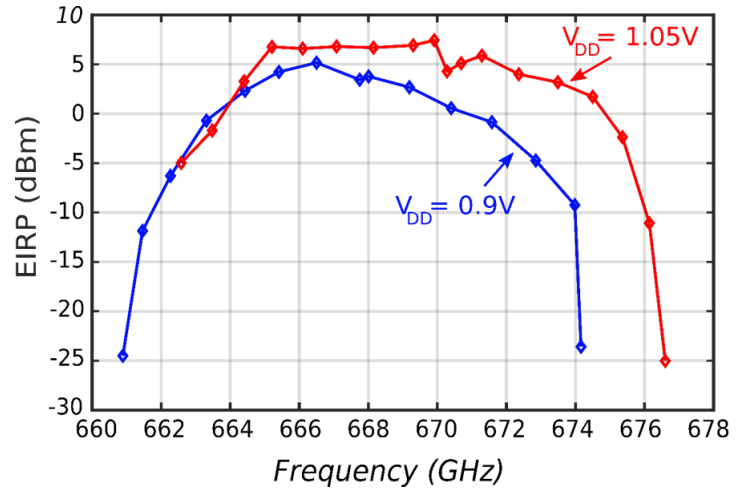

Fig. 11. Measured EIRP over chip tuning range under two different supply values.

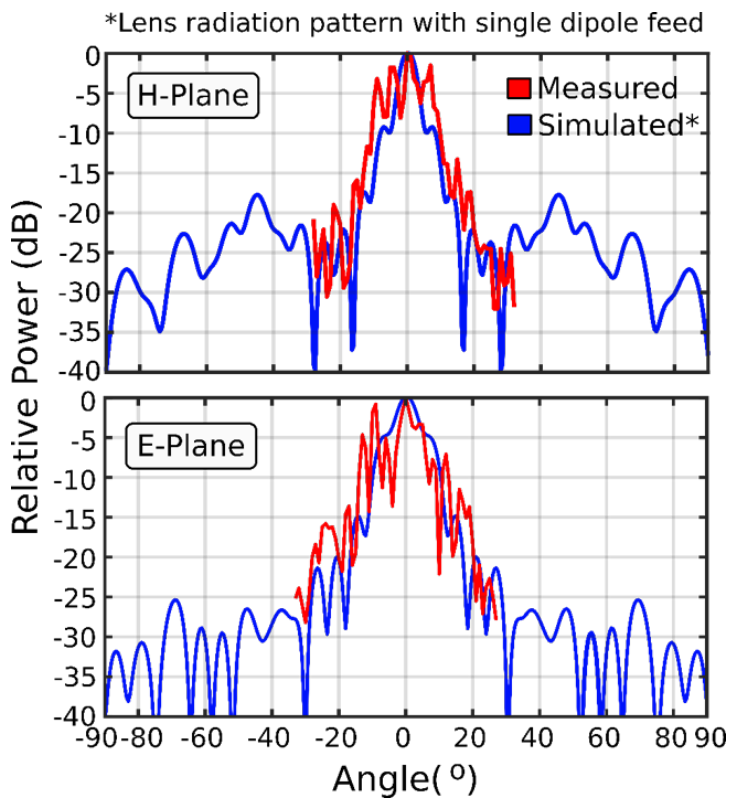

Fig. 12. Measured radiation pattern of $0.67 \mathrm{THz}$ radiator array with substrate attached hyperhemispherical silicon lens.

total radiated power of the chip is dramatically increased by combining the power of numerous radiator elements.

\section{REFERENCES}

[1] F. Friederich et al., "THz active imaging systems with real-time capabilities," IEEE Trans. Terahertz Sci. Tech., vol. 1, no. 1, pp. 183-200, Sept. 2011.

[2] K. Guo and P. Reynaert, "A 475-511GHz radiating source with SIWbased harmonic power extractor in $40 \mathrm{~nm}$ CMOS," IEEE MTT-S Int. Microw. Symp. Dig., Honolulu, HI, 2017, pp. 1-3.

[3] W. Steyaert and P. Reynaert, "A $0.54 \mathrm{THz}$ signal generator in $40 \mathrm{~nm}$ bulk CMOS with $22 \mathrm{GHz}$ tuning range and integrated planar antenna," IEEE J. Solid-State Circuits, vol. 49, no. 7, pp. 1617-1626, July 2014.

[4] Y. Tousi and E. Afshari, "A high-power and scalable 2-D phased array for terahertz CMOS integrated systems," IEEE J. Solid-State Circuits, vol. 50, no. 2, pp. 597-609, Feb. 2015.

[5] K. Guo, Y. Zhang and P. Reynaert, "A 0.53-THz Subharmonic InjectionLocked Phased Array With 63- $\mu$ W Radiated Power in 40-nm CMOS," IEEE J. Solid-State Circuits, vol. 54, no. 2, pp. 380-391, Feb. 2019.

[6] R. Han, C. Jiang, A. Mostajeran, M. Emadi, H. Aghasi, H. Sherry, A. Cathelin, and E. Afshari, "A SiGe terahertz heterodyne imaging 
transmitter with $3.3 \mathrm{~mW}$ radiated power and fully-integrated phaselocked loop," IEEE J. Solid-State Circuits, vol. 50, no. 12, pp. 29352947, Dec. 2015.

[7] Y. Zhao, H.-C. Lu, H.-P. Chen, Y.-T. Chang, R. Huang, H.-N. Chen, C. Jou, F.-L. Hsueh, and M.-C. F. Chang, "A $0.54-0.55 \mathrm{THz} 2 \times 4$ coherent source array with EIRP of $24.4 \mathrm{dBm}$ in $65 \mathrm{~nm}$ CMOS technology," IEEE MTT-S Int. Microw. Symp. Dig., Phoenix, AZ, 2015, pp. 1-3.

[8] Z. Hu and R. Han, "Fully-scalable 2D THz radiating array: A 42-element source in $130-\mathrm{nm} \mathrm{SiGe}$ with $80-\mu \mathrm{W}$ total radiated power at $1.01 \mathrm{THz}$, IEEE Radio Frequency Integrated Circuits Symp., Honolulu, HI, USA, 2017, pp. 372-375.

[9] H. Jalili and O. Momeni, "A 318-to-370GHz standing-wave 2D phased array in $0.13 \mu \mathrm{m}$ BiCMOS," IEEE Int. Solid-State Circuits Conf. Dig. Tech. Papers, San Francisco, CA, 2017, pp. 310-311.

[10] K. Guo and P. Reynaert, "A $0.59 \mathrm{THz}$ beam-steerable coherent radiator array with $1 \mathrm{~mW}$ radiated power and 24.1 EIRP in 40nm CMOS," IEEE Int. Solid-State Circuits Conf. Dig. Tech. Papers, San Francisco, CA, 2020.

[11] G. Guimaraes and P. Reynaert, "A 660 -to- $676 \mathrm{GHz} 4 \times 2$ oscillatorradiator array with intrinsic frequency-filtering feedback for harmonic power boost achieving 7.4dBm EIRP in 40nm CMOS" IEEE Int. SolidState Circuits Conf. Dig. Tech. Papers, San Francisco, CA, 2020.

[12] R. Kananizadeh and O. Momeni, "High-power and high-efficiency millimeter-wave harmonic oscillator design, exploiting harmonic positive feedback in CMOS," IEEE Trans. Microw. Theory Tech., vol. 65, no. 10, pp. 3922-3936, Oct. 2017. 